\title{
Perturbative bosonization from two-point correlation functions
}

\author{
D. Dalmazi, ${ }^{*}$ A. de Souza Dutra ${ }^{\dagger}$ and Marcelo Hott ${ }^{\ddagger}$ \\ UNESP, Campus de Guaratinguetá, DFQ, Av. Dr. Ariberto Pereira da Cunha, 333, CEP 12516-410, Guaratinguetá, SP, Brazil
}

(Received 10 January 2003; published 20 June 2003)

\begin{abstract}
Here we address the problem of bosonizing massive fermions without making expansions in the fermion masses in both massive $\mathrm{QED}_{2}$ and $\mathrm{QED}_{3}$ with $N$ fermion flavors including also a Thirring coupling. We start from two-point correlators involving the $U(1)$ fermionic current and the gauge field. From the tensor structure of those correlators we prove that the $U(1)$ current must be identically conserved (topological) in the corresponding bosonized theory in both $D=2$ and $D=3$ dimensions. We find an effective generating functional in terms of bosonic fields which reproduces these two-point correlators and from that we obtain a map of the Lagrangian density $\bar{\psi}^{r}(i \not b-m) \psi^{r}$ into a bosonic one in both dimensions. This map is nonlocal but it is independent of the electromagnetic and Thirring couplings, at least in the quadratic approximation for the fermionic determinant.
\end{abstract}

DOI: $10.1103 /$ PhysRevD.67.125012

PACS number(s): 11.15.Bt

\section{INTRODUCTION}

One among the many dreams of theoretical physicists nowadays is the possibility of extending to higher dimensions $(D>2)$ the bosonization of fermionic models. This can be justified by some good properties of one formulation as opposed to the other. For instance, strong coupling physics in one model corresponds to weak coupling in the other. A classic example is the map between the massive Thirring and sine-Gordon models in $D=2$. Another interesting aspect of such a map is the fact that the usual electromagnetic charge in the fermionic model corresponds to the topological charge of the associated soliton field. Furthermore, we can have a map between a linear theory such as massive free fermions and a nonlinear one (sine-Gordon model at $\beta^{2}=4 \pi$ ) on the other side.

In view of these and other interesting properties a lot of work has been devoted to the issue of bosonization [1-3] (see also [4]). There have also been many attempts to generalize those ideas to higher dimensions [5-22]. For massive fermions in $D=2$ most of the methods are based on expansions around massless fields that are local conformal theories. In $D=3$, although the case of massless free fermions can still be mapped into a bosonic theory (see [8]) this theory is nonlocal. In addition, the conformal group is finite in $D$ $=3$ and not so powerful as in $D=2$ which makes expansions around the massless case nontrivial. The other possibility is to employ functional methods. Once again the case of massless fermions is easier to deal with since the fermionic determinant can be exactly calculated for $m=0$. For massive fermions in $D=2$ a nontrivial Jacobian under chiral transformations plays a key role in deriving the sine-Gordon model (see [23-27]). In $D=3$, chiral transformations play no role, and although some nonperturbative information is known [21] about the fermionic determinant we are basically

\footnotetext{
*Email address: dalmazi@feg.unesp.br

†Email address: dutra@feg.unesp.br

\#Email address: hott@feg.unesp.br
}

left with approximate methods like the one used in this work. On one hand, the method used here is inspired in the approach carried out in [19], which is rather simple and based on two-point correlation functions. On the other hand, our bosonization rules depart from those in [19] in the sense that they are independent of the interactions. We extend the approach of [19] by introducing an electromagnetic interaction and making use of the $1 / N$ expansion, which allows us to go beyond the lowest order in the coupling constants. In the next section we introduce the model we are working with and obtain a general expression for the generating functional of the current and gauge field correlators. The expression is valid for arbitrary dimensions and depends on the vacuum polarization tensor. In Secs. III and IV we make the calculations explicit in $D=2$ and $D=3$ dimensions, respectively. We first obtain in the fermionic theory the two-point current correlation functions also involving the gauge field, and then we write the current in terms of bosonic fields and derive the corresponding action for such fields that reproduces their correlators. In the final section we draw some conclusions and comment on similar approaches in the literature.

\section{GENERATING FUNCTIONALS}

We start by introducing the notation that will be used in both $D=2$ and $D=3$. The generating functional for a generalized QED with Thirring self-interaction is given by

$$
\begin{aligned}
Z\left[J_{\mu}^{r}, K_{\mu}\right]= & \int \mathcal{D} A_{\mu} \prod_{r=1}^{N} \mathcal{D} \psi_{r} \mathcal{D} \bar{\psi}_{r} \exp i \int d^{D} x\left[-\frac{1}{4} F_{\mu \nu}^{2}\right. \\
& +\bar{\psi}_{r}\left(i \not \partial-m-\frac{e}{\sqrt{N}} A\right) \psi_{r}-\frac{g^{2}}{2 N}\left(\bar{\psi}_{r} \gamma^{\mu} \psi_{r}\right)^{2} \\
& \left.\left.+\frac{\lambda}{2}\left(\partial_{\mu} A^{\mu}\right)^{2}+J_{\mu}^{r}\left(\bar{\psi}_{r} \gamma^{\mu} \psi_{r}\right)+K_{\mu} A^{\mu}\right]\right\}
\end{aligned}
$$

where $N$ is the number of fermion flavors and summation over the repeated flavor index $r(r=1,2, \ldots, N)$ is assumed. 
It is convenient to introduce an auxiliary vector field $B_{\mu}$ and work with the physically equivalent generating functional:

$$
\begin{aligned}
Z\left[J_{\mu}^{r}, K_{\mu}\right]= & \int \mathcal{D} A_{\mu} \mathcal{D} B_{\mu} \prod_{r=1}^{N} \mathcal{D} \psi_{r} \mathcal{D} \bar{\psi}_{r} \\
& \times \exp \left\{i \int d ^ { D } x \left[-\frac{1}{4} F_{\mu \nu}^{2}+\frac{1}{2} B_{\mu} B^{\mu}\right.\right. \\
& +\frac{\lambda}{2}\left(\partial_{\mu} A^{\mu}\right)^{2}+\bar{\psi}_{r}\left(i \not b-m-\frac{e}{\sqrt{N}} \mathbb{A}\right. \\
& \left.\left.\left.-\frac{g}{\sqrt{N}} \boldsymbol{B}+\boldsymbol{J}^{r}\right) \psi_{r}+K_{\mu} A^{\mu}\right]\right\} .
\end{aligned}
$$

Integrating over the fermionic fields we obtain

$$
\begin{aligned}
Z\left[J_{\mu}^{r}, K_{\mu}\right]= & \int \mathcal{D} A_{\mu} \mathcal{D} B_{\mu} \exp \left\{i \int d ^ { n } x \left[-\frac{1}{4} F_{\mu \nu}^{2}+\frac{1}{2} B_{\mu} B^{\mu}\right.\right. \\
& \left.\left.+2 K_{\mu} A^{\mu}+\frac{\lambda}{2}\left(\partial_{\mu} A^{\mu}\right)^{2}\right]\right\} \\
& \times \prod_{r=1}^{N} \operatorname{det}\left[i \not \partial-m-\frac{1}{\sqrt{N}}(e \mathbb{A}+g \not B)+\boldsymbol{J}^{r}\right]
\end{aligned}
$$

Now we compute the fermionic determinant, keeping terms of order $(1 / N)^{0}$ and $(1 / N)^{1 / 2}$. Higher order terms will be neglected. Furthermore, since we are interested only in two-point correlators, the terms higher than quadratic in the sources $J_{\mu}^{r}$ will not be taken into account either. This amounts to the quadratic approximation for the fermionic determinant, which gives

$$
\begin{aligned}
Z\left[J_{\mu}^{r}, K_{\mu}\right]= & \int \mathcal{D} A_{\mu} \mathcal{D} B_{\mu} \exp \frac{l}{2} \int \frac{d^{D} k}{(2 \pi)^{D}} \\
& \times\left\{-\widetilde{A}_{\mu}\left[\theta^{\mu \nu} k^{2}(1-\lambda)+\lambda k^{2} g^{\mu \nu}\right] \widetilde{A}_{\nu}\right. \\
& +\widetilde{B}^{\mu} \widetilde{B}_{\mu}+\sum_{r=1}^{N}\left(e \frac{\widetilde{A}_{\mu}}{\sqrt{N}}+g \frac{\widetilde{B}_{\mu}}{\sqrt{N}}-\widetilde{J}_{\mu}^{r}\right) \\
& \left.\times \Pi^{\mu \nu}\left(k^{2}\right)\left(e \frac{\widetilde{A}_{\nu}}{\sqrt{N}}+g \frac{\widetilde{B}_{\nu}}{\sqrt{N}}-\widetilde{J}_{\nu}^{r}\right)+2 \widetilde{K}_{\mu} \widetilde{A}^{\mu}\right\},
\end{aligned}
$$

where $\theta^{\mu \nu}=g^{\mu \nu}-k^{\mu} k^{\nu} / k^{2}$ and the tildes over the fields represent their Fourier transformations in momentum space. The quantity $\Pi^{\mu \nu}$ is the vacuum polarization tensor:

$$
\Pi^{\mu \nu}(k)=i \int \frac{d^{D} p}{(2 \pi)^{D}} \operatorname{tr}\left[\frac{1}{\not p-m+i \epsilon} \gamma^{\mu} \frac{1}{(\not p+k)-m+i \epsilon} \gamma^{\nu}\right] .
$$

In order to proceed further we have to calculate $\Pi^{\mu \nu}$, which depends on the dimensionality of the space-time.

\section{BOSONIZATION FROM TWO-POINT CORRELATORS IN $D=2$}

In this section we restrict ourselves to the $D=2$ case. Using dimensional regularization we obtain, below the pair creation threshold $\left(z \equiv k^{2} / 4 m^{2}<1\right)$,

$$
\Pi_{\mu \nu}=\widetilde{\Pi}\left(k^{2}\right) \theta_{\mu \nu}
$$

with

$$
\tilde{\Pi}\left(k^{2}\right)=\frac{1}{\pi}\left[1-\frac{1}{[z(1-z)]^{1 / 2}} \arctan \sqrt{\frac{z}{1-z}}\right] . \quad 0<z<1,
$$

$$
\widetilde{\Pi}\left(k^{2}\right)=\frac{1}{\pi}\left[1-\frac{1}{2} \frac{1}{\sqrt{z(z-1)}} \ln \left(\frac{\sqrt{(1-z)}+\sqrt{-z}}{\sqrt{(1-z)}-\sqrt{-z}}\right)\right], \quad z<0 .
$$

Once the tensor $\Pi^{\mu \nu}$ is calculated one is left with a Gaussian integral over the vector fields $A_{\mu}$ and $B_{\mu}$ from which a generating functional quadratic in the sources is derived. This generating functional furnishes the following two-point correlators: ${ }^{1}$

$$
\begin{aligned}
\left\langle j_{\mu}^{r}(k) j_{\nu}^{s}(-k)\right\rangle & =-\frac{\widetilde{\Pi}^{2}\left(e^{2}-k^{2} g^{2}\right)}{N D} \theta^{\mu \nu}+\widetilde{\Pi} \theta^{\mu \nu} \delta^{r s}, \\
\left\langle j_{\mu}^{r}(k) A_{\nu}(-k)\right\rangle & =\frac{e \widetilde{\Pi}}{\sqrt{N} D} \theta^{\mu \nu}, \\
\left\langle A^{\mu}(k) A^{\nu}(-k)\right\rangle & =\left(-\frac{1}{\lambda k^{2}}+\frac{1+g^{2} \tilde{\Pi}}{D}\right) \theta^{\mu \nu}+\frac{g^{\mu \nu}}{\lambda k^{2}},
\end{aligned}
$$

where

$$
D=\left[\widetilde{\Pi} e^{2}-k^{2}\left(1+g^{2} \widetilde{\Pi}\right)\right]
$$

The tensor structure of the above correlation functions is in full agreement with the corresponding Ward identities based on the $U(1)$ symmetry, and it will play a key role in our bosonization procedure. At this point it is important to stress that our approach deviates from that of Ref. [19]. The $1 / N$ expansion we have relied upon, which coincides with the quadratic approximation for the fermionic determinant, is not equivalent to the second order weak coupling expansion used in [19].

\footnotetext{
${ }^{1}$ More precisely, we should have written explicitly the two-point functions in the form $\langle G(k) H(p)\rangle=I(k) \delta^{(2)}(k+p)$ but in this article we will not display the delta function as a matter of convenience. Notice also that when we write $A_{r s}=F+G \delta_{r s}$ it is assumed that $F$ multiplies an $N \times N$ matrix where all entries are equal to 1 .
} 
Now, in order to derive a bosonized expression for the currents $j_{\mu}^{r}=\bar{\psi}_{r} \gamma_{\mu} \psi_{r}$, we write down the most general decomposition for a vector in the momentum space:

$$
j_{\beta}^{r}(k)=\epsilon_{\beta \delta} k^{\delta} \phi^{r}(k)+k_{\beta} \varphi^{r}(k) .
$$

Substituting it in Eq. (9) and using the identity $\epsilon_{\beta \delta} k^{\delta} \epsilon_{\alpha \gamma} k^{\gamma}$ $=k^{2} \theta_{\alpha \beta}$, we obtain

$$
\begin{aligned}
\left\langle j_{\alpha}^{r}(k) j_{\beta}^{s}(-k)\right\rangle= & -k^{2} \theta_{\alpha \beta}\left\langle\phi^{r}(k) \phi^{s}(-k)\right\rangle \\
& -k_{\alpha} k_{\beta}\left\langle\varphi^{r}(k) \varphi^{s}(-k)\right\rangle \\
& -\epsilon_{\alpha \delta^{k}} k^{\delta}\left\langle\phi^{r}(k) \varphi^{s}(-k)\right\rangle \\
& -\epsilon_{\beta \gamma} k^{\gamma} k_{\alpha}\left\langle\varphi^{r}(k) \phi^{s}(-k)\right\rangle \\
= & -\frac{\widetilde{\Pi}^{2}\left(e^{2}-k^{2} g^{2}\right)}{N D} \theta_{\alpha \beta}+\widetilde{\Pi} \theta_{\alpha \beta} \delta^{r s} .
\end{aligned}
$$

From the above it is not difficult to derive

$$
\begin{aligned}
\left\langle\varphi^{r}(k) \varphi^{s}(-k)\right\rangle & =0, \\
\left\langle\phi^{r}(k) \varphi^{s}(-k)\right\rangle & =0, \\
\left\langle\phi^{r}(k) \phi^{s}(-k)\right\rangle & =-\frac{1}{k^{2}}\left[-\frac{\widetilde{\Pi}^{2}\left(e^{2}-k^{2} g^{2}\right)}{N D}+\widetilde{\Pi} \delta^{r s}\right],
\end{aligned}
$$

from which one can safely set $\varphi=0$. On the other hand, substituting the general decomposition

$$
\left\langle\phi(k) A_{\mu}(-k)\right\rangle=M \in_{\mu \delta} k^{\delta}+Q k_{\mu}
$$

in the mixed correlation functions $\left\langle j_{\mu}^{r}(k) A_{\nu}(-k)\right\rangle$ given in Eq. (10), we conclude that

$$
\left\langle\phi^{r}(k) A_{\mu}(-k)\right\rangle=-\frac{e \widetilde{\Pi}}{\sqrt{N} k^{2} D} \epsilon_{\mu \delta} k^{\delta} .
$$

Now we are in a position to derive the bosonic Lagrangian density $\mathcal{L}_{B}\left(A_{\mu}, \phi^{r}\right)$ which is compatible with the correlation functions (11), (17), and (19). For this purpose, we start from the following ansatz:

$$
\begin{aligned}
\mathcal{L}_{B}\left(A_{\mu}, \phi^{r}\right)= & \phi^{r} R_{r s} \phi^{s}+2 S_{r} \phi^{r} \epsilon_{\mu \nu} k^{\nu} A^{\mu} \\
& +A^{\mu} A^{\nu}\left(T_{1} \theta_{\mu \nu}+T_{2} g_{\mu \nu}\right),
\end{aligned}
$$

where $R_{r s}, S_{r}, T_{1}$, and $T_{2}$ are determined as follows. We introduce the external sources $X^{r}$ and $K_{\mu}$ and define the generating functional

$$
\begin{aligned}
Z_{B}\left[X_{r}, K_{\mu}\right]= & \int \prod_{r=1}^{N} \mathcal{D} \phi^{r} \mathcal{D} A_{\mu} \exp \left(i \int d ^ { 2 } x \left[\mathcal{L}_{B}\left(A_{\mu}, \phi^{r}\right)\right.\right. \\
& \left.\left.+X_{r} \phi^{r}+K_{\mu} A^{\mu}\right]\right) .
\end{aligned}
$$

Assuming that $R_{r s}$ is a symmetric nonsingular matrix, we have performed the Gaussian integrals in Eq. (21) and obtained an explicit formula for $Z_{B}\left[X^{r}, K_{\mu}\right]$ from which the two-point correlators can be obtained. By matching these correlators with (11), (17), and (19) we determine the bosonic Lagrangian uniquely:

$$
\begin{aligned}
\mathcal{L}_{B}\left(A_{\mu}, \phi^{r}\right)= & \frac{1}{2}\left[\sum_{r=1}^{N} \phi^{r} \frac{k^{2}}{\widetilde{\Pi}} \phi^{r}+\frac{g^{2}}{N} k^{2}\left(\sum_{r=1}^{N} \phi^{r}\right)\left(\sum_{s=1}^{N} \phi^{s}\right)\right] \\
& -\frac{A^{\alpha} A^{\beta}}{2}\left[(1-\lambda) k^{2} \theta_{\alpha \beta}+\lambda k^{2} g_{\alpha \beta}\right] \\
& +\frac{e}{\sqrt{N}} \epsilon_{\mu \nu} A^{\mu} k^{\nu}\left(\sum_{r=1}^{N} \phi^{r}\right) .
\end{aligned}
$$

All the steps that led us to Eq. (22) are technically simple and not very elucidating. We should mention only that no expansions in either $1 / N$ or any of the coupling constants have been made in those intermediate calculations. Furthermore, notice that if we quantize $\mathcal{L}_{B}\left(A_{\mu}, \phi^{r}\right)$ and integrate over the scalar fields $\phi^{r}$ in Eq. (22) this will lead to a nonlocal effective action for the photon, which was studied in [28], where we concluded that, although nonlocal, the theory is free of tachyons. Next, by comparing with the Lagrangian density written in terms of fermionic fields in Eq. (1) we have the bosonization formulas for each fermion flavor (no sum over repeated roman indices below):

$$
\begin{aligned}
\bar{\psi}_{r} \gamma_{\mu} \psi_{r}(k) & =\epsilon_{\mu \nu} k^{\nu} \phi^{r}(k), \\
-\bar{\psi}_{r}(k)(k+m) \psi_{r}(k) & =\frac{1}{2} \phi^{r} \frac{k^{2}}{\widetilde{\Pi}} \phi^{r} .
\end{aligned}
$$

Now some comments are in order. First of all, if for some given flavor we do a $U(1)$ transformation $\left(\psi_{r} \rightarrow e^{i \alpha} \psi_{r}\right)$ in the expectation value $\left\langle j_{\mu}^{r}\right\rangle$ and use any regularization scheme preserving the $U(1)$ symmetry, it will be easy to derive the Ward identity $\left\langle j_{\mu}^{r} \partial^{\nu} j_{\nu}^{r}\right\rangle=0$ which implies the tensor structure $\left\langle j_{\mu}^{r} j_{\nu}^{r}\right\rangle \propto \theta_{\mu \nu}$, and consequently we will have Eq. (23). So the current is topological due to the $U(1)$ global symmetry and that must hold nonperturbatively. On the other hand, the bosonization rule (24) is only approximate since the full expression would require, in our approach, a complete knowledge of the fermionic determinant, which is possible only for $m \rightarrow 0$. In this case $\widetilde{\Pi} \rightarrow 1 / \pi$ and we end up with $N$ massless scalar fields topologically coupled to the gauge field. Integration over the gauge field leads to $N-1$ massless scalar modes and one mode with $m^{2}=e^{2} /\left(N \pi+g^{2}\right)$, thus reproducing the particular case of the so called SchwingerThirring model for $N=1$ [29], as well as the Schwinger model result $m^{2}=e^{2} / \pi$ for $g \rightarrow 0$ and $N=1$. In the opposite limit of large mass $(z \rightarrow 0)$ we have $\tilde{\pi} \rightarrow-2 z / 3$ at leading order. Substituting back in Eq. (22) we arrive at a divergent result at $m \rightarrow \infty$ which is in agreement with the $m \rightarrow \infty$ limit of the corresponding sine-Gordon model. See [30] for a simi- 
lar comparison in the case of the massive Thirring model without electromagnetic coupling.

\section{BOSONIZATION FROM TWO-POINT CORRELATORS IN $D=\mathbf{3}$}

In $D=3$ dimensions the vacuum polarization tensor (5) calculated by means of dimensional regularization is given by

$$
\Pi^{\mu \nu}(k)=i \Pi_{1} E^{\mu \nu}+\Pi_{2} k^{2} \theta^{\mu \nu},
$$

with $E^{\mu \nu} \equiv \epsilon^{\mu \nu \rho} k_{\rho}$ and, in the range $0 \leqslant z<1$,

$$
\begin{aligned}
& \Pi_{1}=-\frac{1}{8 \pi z^{1 / 2}} \ln \left(\frac{1+z^{1 / 2}}{1-z^{1 / 2}}\right), \\
& \Pi_{2}=\frac{1}{16 \pi m z}\left[1-\left(\frac{1+z}{2 z^{1 / 2}}\right) \ln \left(\frac{1+z^{1 / 2}}{1-z^{1 / 2}}\right)\right],
\end{aligned}
$$

while for $z<0$ we have

$$
\begin{aligned}
& \Pi_{1}=-\frac{1}{4 \pi(-z)^{1 / 2}} \arctan \sqrt{-z}, \\
& \Pi_{2}=\frac{1}{16 \pi m z}\left[1-\left(\frac{1+z}{2(-z)^{1 / 2}}\right) \arctan \sqrt{-z}\right] .
\end{aligned}
$$

In fact the $\Pi_{1}$ amounts to a regularization dependent finite term $[6,7]$, which was taken equal to zero due to the dimensional regularization used.

Substituting Eq. (25) in the general expression (4) we can obtain the two-point functions

$$
\begin{gathered}
\left\langle j_{\mu}^{r}(k) j_{\nu}^{s}(-k)\right\rangle \\
=-\frac{1}{N}\left[k^{2}\left(\Pi_{2}+\frac{P}{\widetilde{Q}}\right) \theta_{\mu \nu}+i \Pi_{1}\left(1-\frac{k^{2}}{\widetilde{Q}}\right) E_{\mu \nu}\right] \\
+\left(k^{2} \Pi_{2} \theta_{\mu \nu}+i \Pi_{1} E_{\mu \nu}\right) \delta_{r s}, \\
\left\langle j_{\mu}^{r}(k) A_{\nu}(-k)\right\rangle=\frac{e}{\widetilde{Q} \sqrt{N}}\left[P \theta_{\mu \nu}-i \Pi_{1} E_{\mu \nu}\right], \\
\left\langle A_{\mu}(k) A_{\nu}(-k)\right\rangle=\frac{g_{\mu \nu}}{\lambda k^{2}}+\left[\frac{e^{2} P}{k^{2} \widetilde{Q}}-\frac{(1+\lambda)}{\lambda k^{2}}\right] \theta_{\nu \mu}-\frac{i e^{2} \Pi_{1}}{k^{2} \widetilde{Q}} E_{\mu \nu},
\end{gathered}
$$

where we found it convenient to define

$$
\begin{aligned}
& P=\left(e^{2}-k^{2} g^{2}\right)\left(k^{2} \Pi_{2}^{2}-\Pi_{1}^{2}\right)-k^{2} \Pi_{2}, \\
& \widetilde{Q}=k^{2}\left[\left(e^{2}-k^{2} g^{2}\right) \Pi_{2}-1\right]^{2}-\left(e^{2}-k^{2} g^{2}\right)^{2} \Pi_{1}^{2} .
\end{aligned}
$$

In analogy with the $D=2$ case, we use for the $U(1)$ current a general decomposition in the momentum space

$$
j_{\alpha}^{r}(k)=\epsilon_{\alpha \beta \gamma} k^{\beta} B_{r}^{\gamma}(k)+k_{\alpha} \phi^{r}(k),
$$

from which we get

$$
\begin{aligned}
\left\langle j_{\alpha}^{r}(k) j_{\beta}^{s}(-k)\right\rangle= & -k_{\alpha} k_{\beta}\left\langle\phi^{r}(k) \phi^{s}(-k)\right\rangle \\
& +E_{\alpha \gamma} E_{\delta \beta}\left\langle B_{r}^{\gamma}(k) B_{s}^{\delta}(-k)\right\rangle \\
& +k_{\alpha} E_{\beta \delta}\left\langle\phi^{r}(k) B_{s}^{\delta}(-k)\right\rangle \\
& +k_{\beta} E_{\alpha \gamma}\left\langle B_{r}^{\gamma}(k) \phi_{s}(-k)\right\rangle .
\end{aligned}
$$

Multiplying the last expression by $k^{\alpha} k^{\beta}$, we conclude that $\left\langle\phi^{r}(k) \phi^{s}(-k)\right\rangle=0$. Now, multiplying the resulting expression by $k^{\alpha}$, we have $E_{\alpha \beta}\left\langle\phi^{r}(k) B_{s}^{\beta}(-k)\right\rangle=0$. A similar manipulation was used in the last section to derive Eqs. (15), (16), and (17). Concluding, we can certainly neglect the scalar fields $\phi^{r}=0$ and minimally bosonize the $U(1)$ current in $D=3$ in terms of a bosonic vector field. The bosonic version of the current is once again of topological nature and identically conserved. As in the $D=2$ case, this happens because of the $U(1)$ global symmetry of the fermionic Lagrangian. Taking $\phi^{r}=0$ and substituting the decomposition (33) in Eqs. (28) and (29), after some trivial manipulations we end up with

$$
\left\langle B_{r}^{\gamma}(k) B_{s}^{\delta}(-k)\right\rangle=-C_{r s} g^{\gamma \delta}+\left(C-\frac{H_{2}}{k^{2}}\right)_{r s} \theta^{\gamma \delta}-\frac{\left(H_{1}\right)_{r s}}{k^{2}} E^{\gamma \delta},
$$

$$
\begin{aligned}
\left\langle A^{\nu}(k) B_{s}^{\mu}(-k)\right\rangle= & -\left(\frac{i e \Pi_{1}}{\sqrt{N} \widetilde{Q}}+D\right)_{s} g^{\nu \mu}-\left(\frac{e P}{\sqrt{N} \widetilde{Q} k^{2}}\right)_{s} E^{\nu \mu} \\
& +D_{s} \theta^{\nu \mu}
\end{aligned}
$$

where $C_{r s}$ and $D_{s}$ are arbitrary and

$$
\begin{aligned}
& \left(H_{1}\right)_{r s}=i \Pi_{1} \delta_{r s}+\frac{i \Pi_{1}}{N}\left(\frac{k^{2}}{\widetilde{Q}}-1\right), \\
& \left(H_{2}\right)_{r s}=k^{2} \Pi_{2} \delta_{r s}-\frac{k^{2}}{N}\left(\Pi_{2}+\frac{P}{\widetilde{Q}}\right) .
\end{aligned}
$$

The arbitrariness of $C_{r s}$ and $D_{s}$ shows that the bosonization fields $B_{r}^{\mu}$ must be gauge fields, but the corresponding gauge symmetry is independent of the electromagnetic one. Analogous to the last section, we next derive a Lagrangian density compatible with the two-point correlators (35), (36), and (30). Let us suppose we have a bosonic Lagrangian density $\mathcal{L}_{B}\left(A_{\mu}, B_{r}^{\nu}\right)$ of the form

$$
\begin{aligned}
\mathcal{L}_{B}\left(A_{\mu}, B_{r}^{\nu}\right)= & B_{r}^{\mu} \mathcal{O}_{\mu \nu}^{r s} B_{s}^{\nu}+u^{s} B_{s}^{\mu} E_{\mu \nu} A^{\nu} \\
& +A_{\mu} A_{\nu}\left(v_{1} \theta_{\mu \nu}+v_{2} g_{\mu \nu}\right),
\end{aligned}
$$

where $\mathcal{O}_{\mu \nu}^{r s}$ has a general tensor structure in both momentum and flavor space,

$$
\mathcal{O}_{\mu \nu}^{r s}=a^{r s} \theta_{\mu \nu}+b^{r s} E_{\mu \nu}+d^{r s} g_{\mu \nu} .
$$


The quantities $a^{r s}, b^{r s}, d^{r s}, u^{s}, v_{1}, v_{2}$ are determined by matching the two-point correlators derived from the generating functional

$$
\begin{aligned}
Z_{B}\left[Y_{\nu}^{r}, K_{\mu}\right]= & \int \prod_{r=1}^{N} \mathcal{D} B_{r}^{\mu} \mathcal{D} A_{\mu} \exp \left(i \int d ^ { 2 } x \left[\mathcal{L}_{B}\left(A_{\mu}, B_{r}^{\nu}\right)\right.\right. \\
& \left.\left.+Y_{\mu}^{r} B_{r}^{\mu}+K_{\mu} A^{\mu}\right]\right)
\end{aligned}
$$

with the correlators (35), (36), and (30). Assuming that the matrices $a^{r s}, b^{r s}, d^{r s}$ are symmetric and nonsingular, our first step is to perform the Gaussian integrals over the $N$ vector fields $B_{r}^{\nu}$, which furnishes

$$
\begin{aligned}
Z_{B}\left[Y_{\nu}^{r}, K_{\mu}\right]= & \int \mathcal{D} A_{\mu} \exp \left\{i \int d ^ { 2 } x \left[-\frac{1}{4}\left(Y_{r}^{\mu}+u_{r} A_{\beta} E^{\beta \mu}\right)\right.\right. \\
& \times\left(\mathcal{O}^{-1}\right)_{\mu \nu}^{r s}\left(Y_{s}^{\nu}+u_{s} E^{\nu \beta} A_{\beta}\right) \\
& \left.\left.+A^{\mu} A^{\nu}\left(v_{1} \theta_{\mu \nu}+v_{2} g_{\mu \nu}\right)+K_{\mu} A^{\mu}\right]\right\}
\end{aligned}
$$

The inverse operator $\mathcal{O}^{-1}$ has the same structure as Eq. (40): $\left(\mathcal{O}^{-1}\right)_{\mu \nu}^{r s}=\tilde{a}^{r s} \theta_{\mu \nu}+\widetilde{b}^{r s} E_{\mu \nu}+\widetilde{d}^{r s} g_{\mu \nu}$, where the tilde variables are functions of the nontilde ones. In particular, $\widetilde{d}_{r s}$ $=\left(d^{-1}\right)_{r s}$. Since we have the contractions $E^{\mu \nu} E_{\nu \alpha}=$ $-k^{2} \theta_{\alpha}^{\mu}, E^{\mu \nu} \theta_{\nu \alpha}=E_{\alpha}^{\mu}$, it becomes clear from Eq. (42), with a little thought, that even after integrating over $A_{\mu}$ the only term in $\log Z_{B}$ quadratic in the sources $Y_{r}^{\mu}$ and contracted via the metric tensor is $-\frac{1}{4} Y_{r}^{\mu} Y_{s}^{\nu} \widetilde{d}^{r s} g_{\mu \nu}$. Therefore the twopoint functions (35) require the identification $C^{r s}=(\widetilde{d} / 2)^{r s}$ $=\left(d^{-1} / 2\right)^{r s}$.

Given that the matrix $C^{r s}$ is arbitrary, in order to simplify the calculations, we have chosen it proportional to the identity, and consequently we reduce the undetermined parameters in our original ansatz for the bosonic Lagrangian since $d^{r s}=d \delta^{r s}$. For analogous reasons, after integrating over $A_{\mu}$, the crossed terms in the sources $Y_{r}^{\mu}$ and $K^{\nu}$ are contracted only by the tensors $E_{\mu \nu}$ and $\theta_{\mu \nu}$. Thus, the term proportional to $g^{\nu \mu}$ on the right hand side of the correlation functions (36) must vanish, which fixes $D=-i e \Pi_{1} / \sqrt{N} \widetilde{Q}$. Finally, comparing the other parts of the correlators (35), (36), and (30) with the final expression for $\log Z_{B}$ one can determine the quantities $a^{r s}, b^{r s}$, and $u^{s}$ as functions of $d$, which remains unfixed. We spare the reader the lengthy details since they are totally technical and not very illuminating. We stress only that no further simplification hypothesis is used and no expansion is made in either $1 / N$ or any of the coupling constants. In those intermediate steps all correlators are treated as if they were exact.

In order to make the final answer for the bosonic Lagrangian density more familiar, we have redefined the unfixed pa- rameter $d \equiv-\tilde{\lambda} k^{2} / 2$. In the momentum space we have

$$
\begin{aligned}
\mathcal{L}_{B}\left(A_{\mu}, B_{r}^{\alpha}\right)= & -\frac{1}{2}\left[\sum_{r=1}^{N} \frac{B_{r}^{\alpha}\left(k^{2} \Pi_{2} \theta_{\alpha \beta}+i \Pi_{1} \epsilon_{\alpha \beta \gamma} k^{\gamma}\right) B_{r}^{\beta}}{k^{2} \Pi_{2}^{2}-\Pi_{1}^{2}}\right. \\
& \left.+\frac{g^{2}}{N}\left(\sum_{r=1}^{N} B_{r}^{\alpha}\right) k^{2} \theta_{\alpha \beta}\left(\sum_{s=1}^{N} B_{s}^{\beta}\right)\right] \\
& -\frac{A^{\alpha} A^{\beta}}{2}\left[(1-\lambda) k^{2} \theta_{\alpha \beta}+\lambda k^{2} g_{\alpha \beta}\right] \\
& +\frac{e}{\sqrt{N}} \epsilon_{\mu \nu \gamma} k^{\gamma} A^{\mu} \sum_{r=1}^{N} B_{r}^{\nu} \\
& +\sum_{r=1}^{N} \frac{B_{r}^{\alpha} B_{r}^{\beta}}{2}\left[\tilde{\lambda} k^{2} \theta_{\alpha \beta}-\tilde{\lambda} k^{2} g_{\alpha \beta}\right] .
\end{aligned}
$$

Notice that $\lambda$ and $\tilde{\lambda}$ are interpreted as independent gauge fixing parameters such that the generating functional (41) reproduces the correlation functions (30), (35), and (36) with

$$
\begin{gathered}
C_{r s}=-\frac{1}{\widetilde{\lambda} k^{2}} \delta_{r s}, \\
D=-\frac{i e \Pi_{1}}{\sqrt{N} \widetilde{Q}} .
\end{gathered}
$$

Integration over the vector fields $B_{r}^{\alpha}$ will lead to an effective electromagnetic theory, which was also studied in [28] (see also [23]), where we analyzed its pole structure and concluded that no tachyons appear, just as in its two-dimensional counterpart (22).

Comparing Eq. (43) with the original Lagrangian (1) written in terms of fermionic fields, we have the bosonization rules for each fermion flavor $(r=1,2, \ldots, N)$ :

$$
\begin{aligned}
\bar{\psi}_{r} \gamma_{\mu} \psi_{r}(k) & =\epsilon_{\mu \nu \alpha} k^{\nu} B_{r}^{\alpha}(k), \\
-\bar{\psi}_{r}(k)(k+m) \psi_{r}(k) & =-\frac{1}{2} B_{r}^{\alpha} \frac{\left(k^{2} \Pi_{2} \theta_{\alpha \beta}+i \Pi_{1} E_{\alpha \beta}\right)}{k^{2} \Pi_{2}^{2}-\Pi_{1}^{2}} B_{r}^{\beta} .
\end{aligned}
$$

As in the $D=2$ case, we obtained Eq. (46) from the fact that for each fermion flavor we have the tensor structure $\left\langle j_{\mu} j_{\nu}\right\rangle \propto a \theta_{\mu \nu}+b E_{\mu \nu}$, which was derived by calculating the fermionic determinant up to quadratic order. However, this tensor structure must hold beyond that approximation (nonperturbatively) as a consequence of the Ward identity $\left\langle j_{\mu} \partial^{\nu} j_{\nu}\right\rangle=0$, which must be true also in $D=3$, by using a gauge invariant regularization. On the other hand, the bosonization rule (47) holds as a consequence of the quadratic approximation for the fermionic determinant. Taking $m \rightarrow 0$ (or $z \rightarrow-\infty)$ in the expressions (27) we have $\Pi_{1} \rightarrow 0$ and $\Pi_{2} \rightarrow 1 /\left(16 \sqrt{-k^{2}}\right)$. Plugging these values back in Eqs. (46) and (47), we recover the result of [8] with $\beta=1 / 4$ and $\theta=0$ in that reference, which deals with massless fermions. 
Our rules (46) and (47) also agree with the case of massive fermions treated in [15]. We stress, however, that in both [8] and [15] only free fermions were considered, while our results were derived for an interacting theory with Thirring and electromagnetic couplings. This indicates that our bosonization rules are rather universal at the quadratic level. In the opposite limit of heavy fermions $\left(k^{2} / 4 m^{2}\right)=z \rightarrow 0$ in Eq. (26) we have, at leading order, $\Pi_{2} \rightarrow 0$ and $\Pi_{1} \rightarrow-1 /(4 \pi)$ which leaves us [see Eq. (47)], with a bosonic local and topological Chern-Simons Lagrangian for the field $B_{r}^{\mu}$, in agreement with the findings of [10] (see also [11] and [12] for higher orders).

\section{CONCLUSIONS}

We derived bosonization maps for the $U(1)$ currents and the fermion Lagrangian densities for both $\mathrm{QED}_{2}$ and $\mathrm{QED}_{3}$ with $N$ fermion flavors and Thirring self-interaction. Both results hold for finite fermion masses and no derivative expansion in $\mathrm{k} / \mathrm{m}$ is made as in [31]. By turning off the interactions we can reproduce the results of [15] for a particular choice of the regularization parameter in that reference. Our calculations show that the $U(1)$ currents, when written in terms of bosons, must be identically conserved (topological) as a direct consequence of the Ward identity $\left\langle j_{\mu} \partial^{\nu} j_{\nu}\right\rangle=0$, which is true nonperturbatively if there is no $U(1)$ anomaly. In particular, there is no need to look at higher point functions or compute the fermionic determinant beyond the quadratic approximation to confirm that. On the other hand, our maps for the Lagrangian densities (24) and (47), which are also independent of the interactions, hold only perturbatively due to our quadratic approximation for the fermionic determinant, which amounts to neglecting terms of order higher than $(1 / N)^{1 / 2}$ in the fermionic determinant and considering at most two-point current correlators to derive the bosonization rules.

Finally, we should mention that the results derived here could have been obtained in a technically more direct way along the lines of [20] (see also [32]) but the approach used here does not use any auxiliary field and clarifies the fundamental role of the two-point correlators. It might also be useful (work in progress) for deriving approximate bosonic maps for other fermion bilinears, like the mass term $\bar{\psi} \psi$ in $D=3$, which apparently could not be done using the approach of $[20]$.

\section{ACKNOWLEDGMENTS}

This work was partially supported by CNPq and FAPESP, Brazilian research agencies.
[1] S. Coleman, R. Jackiw, and L. Susskind, Ann. Phys. (N.Y.) 93, 267 (1975).

[2] S. Coleman, Phys. Rev. D 11, 2088 (1975); Ann. Phys. (N.Y.) 101, 239 (1976).

[3] S. Mandelstam, Phys. Rev. D 11, 3026 (1975).

[4] E. Abdalla, M.C. Abdalla, and K.D. Rothe, Non-Perturbative Methods in Two Dimensional Quantum Field Theory (World Scientific, Singapore, 1991).

[5] A.M. Polyakov, in Field Theory and Critical Phenomena, 1988 Les Houches Lectures, edited by E. Brézin and J. ZinnJustin (Elsevier, Amsterdam, 1989).

[6] A. Coste and M. Luscher, Nucl. Phys. B323, 631 (1989).

[7] M. Luscher, Nucl. Phys. B326, 557 (1989).

[8] E.C. Marino, Phys. Lett. B 263, 63 (1991).

[9] C.P. Burgess, C. Lutken, and F. Quevedo, Phys. Lett. B 336, 18 (1994).

[10] E. Fradkin and F.A. Schaposnik, Phys. Lett. B 338, 253 (1994); G. Rossini and F.A. Schaposnik, ibid. 338, 465 (1994).

[11] R. Banerjee, Phys. Lett. B 358, 297 (1995).

[12] R. Banerjee, Nucl. Phys. B465, 157 (1996).

[13] F.A. Schaposnik, Phys. Lett. B 356, 39 (1995).

[14] N. Bralic, E. Fradkin, V. Manias, and F.A. Schaposnik, Nucl. Phys. B446, 144 (1995).

[15] D.G. Barci, C.D. Fosco, and L.E. Oxman, Phys. Lett. B 375, 367 (1996).

[16] N. Banerjee, R. Banerjee, and S. Ghosh, Nucl. Phys. B481, 421 (1996).
[17] J.C. Le Guillou, C. Núñez, and F.A. Schaposnik, Ann. Phys. (N.Y.) 251, 426 (1996).

[18] R. Banerjee and E.C. Marino, Phys. Rev. D 56, 3763 (1997).

[19] R. Banerjee and E.C. Marino, Nucl. Phys. B507, 501 (1997).

[20] D.G. Barci, L.E. Oxman, and S.P. Sorella, Phys. Rev. D 59, 105012 (1999).

[21] D.G. Barci, V.E.R. Lemes, C. Linhares de Jesus, M.B.D. Silva M. Porto, S.P. Sorella, and L.C.Q. Vilar, Nucl. Phys. B524, 765 (1998).

[22] A. de Souza Dutra and C.P. Natividade, Mod. Phys. Lett. A 14, 307 (1999); Phys. Rev. D 61, 027701 (2000).

[23] D. Dalmazi, A. de Souza Dutra, and M. Hott, Phys. Rev. D 61, 125018 (2000).

[24] K. Furuya, R.E. Gamboa-Saravi, and F.A. Schaposnik, Nucl. Phys. B208, 159 (1982).

[25] C.M. Naon, Phys. Rev. D 31, 2035 (1985).

[26] L.C.L. Botelho, Phys. Rev. D 33, 1195 (1986).

[27] P.H. Damgaard, H.B. Nielsen, and R. Sollacher, Nucl. Phys. B385, 227 (1992); B296, 132 (1992).

[28] E.M.C. Abreu, D. Dalmazi, A. de Souza Dutra, and M. Hott, Phys. Rev. D 65, 125030 (2002).

[29] A. de Souza Dutra, C.P. Natividade, H. Boschi-Filho, R.L.P.G. Amaral, and L.V. Belvedere, Phys. Rev. D 55, 4931 (1997).

[30] K-I. Kondo, Prog. Theor. Phys. 94, 899 (1995).

[31] S. Ghosh, "Bosonization and Duality in 2+1 Dimensions: Applications in Gauged Massive Thirring Model," hep-th/9901051.

[32] C.P. Burgess and F. Quevedo, Nucl. Phys. B421, 373 (1994). 